\title{
Fleas as Vectors of Especially Dangerous Infections in Jermuk Region, 2010-2016
}

\section{Lusine Markosyan* and Vahe Kulakhszyan}

Laboratory of Epizootology, Ectoparasitology and Entomology, RA MOH National Center for Disease Control and Prevention SNCO, Reference Laboratory Center branch, Yerevan, Armenia

\section{Objective}

The goal was to determine the impact of flea number variation on the epizootic situation in the Jermuk region.

\section{Introduction}

The Jermuk region of the Zangezur mezofocus is part of the transcaucasian highland focus of plague. This enzootic area is polyvectorial. The mezofocus has rich fauna with approximately eight species of fleas: Callopsylla caspia, Ctenophthalmus wladimiri, Frontopsylla elata, Amphipsylla rossica, Leptopsylla taschenbergi, Nosopsyllus consimilis, Palaeopsylla vartanovi, and Doratopsylla dampfi. Ct. wladimiri is the most abundant. However, special attention should be paid to C. caspia and N. consimilis as they are the only vectors specific for Yersinia pestis. In these fleas, the bacteria form a plug that blocks digestion and induces starvation. Afflicted fleas bite frenziedly in an effort to feed and the pressure that results releases bacteria from the plug, infecting a new host. Fleas infected with plague during an epizootic are a serious threat to humans, especially when in contact with synanthropic rodents. A survey was conducted to catalog fleas in the foci.

\section{Methods}

From 2010-2016 different species of fleas were collected in Jermuk region of Vayots Dzor by combing the hair of captured rodents and processing their nests with heat and light. Flea species, and their egglaying status, were identified by microscopic examination.

\section{Results}

From 2010-2015 the density of C. caspia averaged 23 per hectare. Fleas that were laying eggs were quantified via microscopy. In 2016 a drastic increase in C. caspia density was recorded; flea numbers averaged 225 per hectare. Half of these were actively reproducing (Table 1). These changes in flea numbers occurred with a stable rodent population of 60 per hectare.

\section{Conclusions}

Because of the drastic increase in flea density of 2016 compared to the period of 2010-2015, it is likely that diseases that depend on fleas to spread will increase in the near future in Jermuk. So, it is necessary to monitor the epizootic situation of Jermuk, as it is a popular resort in Armenia.

C. caspia numbers and fertility in Jermuk

\begin{tabular}{|c|c|c|}
\hline Year & Fleas per hectare & \% of egg-laying fleas per hectare \\
\hline 2010 & 67 & $70 \%$ \\
\hline 2011 & 13 & $79 \%$ \\
\hline 2012 & 15 & $55 \%$ \\
\hline 2013 & 8 & $52 \%$ \\
\hline 2014 & 10 & $53 \%$ \\
\hline 2015 & 27 & $18 \%$ \\
\hline 2016 & 225 & $50 \%$ \\
\hline
\end{tabular}

Keywords

Callopsylla caspia; fleas; population survey

*Lusine Markosyan

E-mail: lusine.markosyan.1976@mail.ru 